\title{
Noncoherent Physical-Layer Network Coding Using Binary CPFSK Modulation
}

\author{
Matthew C. Valenti*, Don Torrieri ${ }^{\dagger}$ and Terry Ferrett* \\ *West Virginia University, Morgantown, WV, USA. \\ ${ }^{\dagger}$ U.S. Army Research Laboratory, Adelphi, MD, USA.
}

\begin{abstract}
Physical-layer network coding is a high-throughput technique for communicating over the two-way relay channel, which consists of two terminals that communicate exclusively via an intermediate relay. An exchange of messages begins with both terminals transmitting binary data sequences simultaneously to the relay. The relay determines the modulo-2 sum of the sequences, which it modulates and broadcasts to the terminals. Since each terminal knows the information it transmitted, it can determine the information transmitted by the other terminal by subtracting its own information from the broadcast signal. Prior work on the topic of physical-layer network coding has assumed that the signals transmitted by the two terminals arrive at the relay with perfectly aligned phases, permitting coherent reception. In this paper, we relax the assumption of aligned phases and consider noncoherent reception of binary continuous-phase frequency-shift keying signals. A derivation of the relay receiver is given for varying amounts of channel state information, and results are provided showing the error performance of the proposed system without an outer errorcorrecting code and with an outer turbo code.
\end{abstract}

\section{INTRODUCTION}

Network coding is a technique introduced by Ahlswede et. al. [1] to improve throughput by encoding information at intermediate nodes in a multi-hop network. Encoding is performed at a relay by combining information received from multiple input links, and forwarding the combined information. This technique is in contrast to the traditional switching mechanism performed by relays, in which every received input is forwarded separately. Thus, network coding reduces the required number of forwarding operations in a network, thereby increasing throughput at the expense of more complex decoding at each destination.

The smallest network for which network coding may be advantageously applied is the two-way relay channel, which is a three-terminal network with two end nodes $N_{1}$ and $N_{2}$ that wish to exchange messages and an intermediate relay $R$. In this paper, we assume that all three terminals operate under a half-duplex constraint and there is no direct link between the two end nodes. Thus, all communication must flow through the relay. With conventional network coding, communication over the two-way relay channel occurs in two phases, a multipleaccess phase and a broadcast phase. During the multipleaccess phase, the two end-nodes communicate using a standard multiple-access scheme, such as time-division multiple access (TDMA). In the broadcast phase, the modulo- 2 sum of the

M.C. Valenti and T. Ferrett were supported in part by the National Science Foundation under Award No. CNS-0750821.

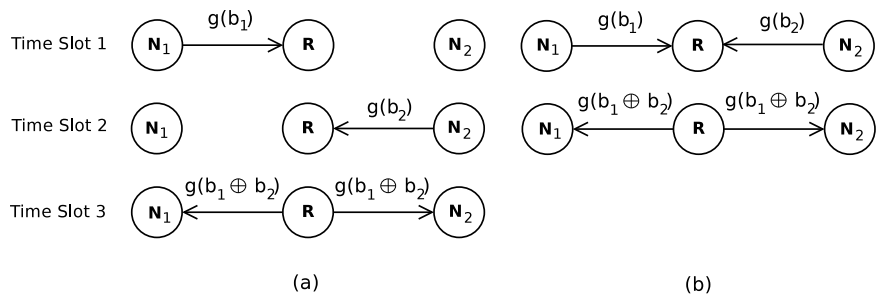

Fig. 1. (a) Link-layer network coding, and (b) Physical-layer network coding.

messages received at the relay are broadcast to the two end nodes, assuming the modulation was binary.

Fig. 1(a) shows an example using TDMA. Let $b_{i}$ be node $N_{i}$ 's message bit and let $g(\cdot)$ be a modulation function. $N_{1}$ transmits its modulated information, $g\left(b_{1}\right)$, to $R$ in the first TDMA time slot. $N_{2}$ transmits its modulated information, $g\left(b_{2}\right)$, in the second TDMA time slot. Relay $R$ demodulates the two bits, encodes them by adding them modulo-2 ( $\left.b=b_{1} \oplus b_{2}\right)$, and broadcasts the encoded and modulated bit $g\left(b_{1} \oplus b_{2}\right)$ to $N_{1}$ and $N_{2}$ in a third time slot (the broadcast slot). Each end node $N_{i}$ demodulates the broadcast to reveal an estimate of $b$ and then determines the information from the other terminal by adding its own information to $b$ modulo-2 (e.g., $N_{1}$ determines $N_{2}$ 's information by using $b_{2}=b_{1} \oplus b$ ). To distinguish it from physical-layer network coding, which is discussed below, we refer to this traditional network coding technique as link-layer network coding (LNC).

Physical-layer network coding (PNC) [2], [3] is an efficient alternative to LNC. The schedule for PNC is illustrated in Fig. 1(b). With PNC, both end nodes transmit during the first slot and their signals are added at the receive antenna prior to demodulation. This is in contrast with LNC, which involves the addition of information at the relay after demodulation. A properly designed relay receiver should translate the received signal into the same combined data signal that it would have computed had LNC been used. This technique yields an even greater throughput gain than the LNC scheme described above because simultaneous transmission by $N_{1}$ and $N_{2}$ in the first time slot reduces the required number of time slots from three to two.

A common assumption made in the previous literature is that the phases of the signals received by the relay are synchronized and that reception is coherent. For instance, decodeand-forward relaying has been considered for binary phaseshift keying [4] and minimum-shift keying [5] modulations, but in both cases the relay must perform coherent reception. 
An amplify-and-forward protocol is considered in [6], which allows the decision to be deferred by the relay to the end-node, though detection is still coherent.

When two signals arrive concurrently at a common receiver, neither coherent detection nor the cophasing of the two signals (so that they arrive with a constant phase offset) is practical. The latter would require preambles that detract from the overall throughput, stable phases, and small frequency mismatches. In this paper, physical-layer network coding is achieved with binary continuous-phase frequency-shift keying (CPFSK) modulation, which permits noncoherent reception. While our motivation for using noncoherent CPFSK is its application to PNC, noncoherent CPFSK is a good choice even for conventional point-to-point links and for LNC. This is because CPFSK has a constant envelope, thereby permitting efficient amplification, and the continuous-phase constraint provides a compact spectrum. Furthermore, noncoherent demodulation is often required when the time that a packet dwells at a particular frequency is too short to permit acquisition of the carrier, which is a characteristic of frequencyhopping systems. These characteristics make CPFSK a popular choice for military communications, and therefore we assume the use of CPFSK even when considering the LNC system.

The main contribution of this paper is the derivation of a relay receiver that performs noncoherent reception and does not require phase synchronism. Several receiver formulations are given for the relay receiver that differ by the required amount of channel state information. Simulation results are provided both with and without an outer turbo code. When the turbo code is used in the proposed noncoherent PNC system, a throughput gain of $20 \%$ over the comparable turbo-coded LNC system is possible when the relay receiver only knows the fading statistics, and a gain of nearly $50 \%$ is possible when the relay knows the actual fading amplitudes.

\section{SYSTEM MODEL}

Consider the three terminal network shown in Fig. 1. The network contains a pair of end nodes, $N_{i}, i \in\{1,2\}$, and a relay $R$. Each of the end nodes encodes a separate length$K$ binary message $\mathbf{u}_{i}$ using a rate- $r$ linear binary encoder. The output of node $N_{i}$ 's encoder is the length $L=K / r$ codeword $\mathbf{b}_{i}$. Each node transmits its codeword to the relay using binary orthogonal frequency-shift keying (FSK). With link-layer network coding (LNC), the two nodes transmit during two orthogonal time slots, and thus their transmitted signals do not interfere. However, with physical-layer network coding (PNC), both nodes transmit simultaneously.

Let $b_{i} \in\{0,1\}$ be the code bit transmitted by node $N_{i}$ during a particular signaling interval. The complex envelope of node $N_{i}$ 's modulated signal $x_{i}(t)$ is chosen as the $b_{i}^{t h}$ signal of the set of continuous-time signals $\mathcal{S}=\left\{s_{k}(t), k=0,1\right\}$, where

$$
s_{k}(t)=\frac{1}{\sqrt{T_{s}}} e^{\frac{j 2 \pi k h t}{T_{s}}}, \quad t \in\left[0, T_{s}\right),
$$

$h$ is the modulation index, and $T_{s}$ is the symbol duration. To ensure that the two possible transmitted tones are orthogonal under noncoherent detection, the modulation index $h$ must be an integer, and in this paper we assume $h=1$. For purposes of controlling the spectrum, a continuous phase transition from one symbol period to the next is required. When the latter two requirements are satisfied, the modulation is an instance of continuous-phase frequency shift keying (CPFSK) [6].

The signal transmitted by each node is sent to the relay over a flat-fading channel. Let $h_{i}$ represent the complex-valued channel gain from node $N_{i}$ to the relay during the signaling interval. The gain may be represented as $h_{i}=\alpha_{i} e^{j \theta_{i}}$, where $\alpha_{i}$ is the received amplitude and $\theta_{i}$ is the phase shift due to the fading, the transmitter's continuous-phase constraint, and the offset between the transmitter's oscillator and receiver's oscillator. Because the energies of the transmitted signals are normalized to unity, the power of the channel gains are selected such that the energy received by the relay from node $N_{i}$ is $\mathcal{E}_{i}$, where

$$
\mathcal{E}_{i}=E\left[\left|h_{i}\right|^{2}\right]=E\left[\alpha_{i}^{2}\right] .
$$

For the LNC system, the complex envelope of the signal received by the relay is

$$
y(t)=h_{i} x_{i}(t)+n(t),
$$

where $n(t)$ is additive white Gaussian noise (AWGN) with two-sided noise spectral density $N_{0}$, and the index $i=1$ or 2 depending on whether node $N_{1}$ or node $N_{2}$ was transmitting during the particular time slot.

The front-end of the detector is a bank of two pairs of matched filters, with one pair matched to the in-phase and quadrature components of each tone in $\mathcal{S}$. The matched filters are sampled at the symbol rate and the output is placed into the $2 \times 1$ complex vector

$$
\mathbf{y}=h_{i} \mathbf{x}_{i}+\mathbf{n}
$$

where $\mathbf{x}_{i}$ is a signal vector, $\mathbf{n}$ is zero-mean circularlysymmetric complex Gaussian noise with covariance matrix $N_{0} \mathbf{I}_{2}$, and $\mathbf{I}_{2}$ is the 2-by-2 identity matrix. Since $h=1$, the signal vector may be represented by

$$
\mathbf{x}_{i}=\left[\begin{array}{ll}
\left(1-b_{i}\right) & b_{i}
\end{array}\right]^{T} .
$$

For the PNC system, both end nodes transmit at the same time in the same time slot. During a particular signaling interval the relay receives

$$
y(t)=h_{1} x_{1}(t)+h_{2} x_{2}(t)+n(t),
$$

the output of the bank of matched filters is represented by the vector

$$
\mathbf{y}=h_{1} \mathbf{x}_{1}+h_{2} \mathbf{x}_{2}+\mathbf{n},
$$

and the zero-mean circularly-symmetric complex Gaussian noise $\mathbf{n}$ again has covariance matrix $N_{0} \mathbf{I}_{2}$.

Define the following four events:

1) Event $\mathbb{E}_{1}=\left\{b_{1}=0, b_{2}=0\right\}$

2) Event $\mathbb{E}_{2}=\left\{b_{1}=1, b_{2}=1\right\}$

3) Event $\mathbb{E}_{3}=\left\{b_{1}=0, b_{2}=1\right\}$ 
4) Event $\mathbb{E}_{4}=\left\{b_{1}=1, b_{2}=0\right\}$.

These events represent the possible combinations of transmitted bits during one signaling interval. Given event $\mathbb{E}_{i}$, the signal received by the relay in the PNC system is

$$
\mathbf{y}=\mathbf{m}_{i}+\mathbf{n}
$$

where

$$
\begin{aligned}
& \mathbf{m}_{1}=\left[\begin{array}{ll}
\left(h_{1}+h_{2}\right) & 0
\end{array}\right]^{T} \\
& \mathbf{m}_{2}=\left[\begin{array}{ll}
0 & \left(h_{1}+h_{2}\right)
\end{array}\right]^{T} \\
& \mathbf{m}_{3}=\left[\begin{array}{ll}
h_{1} & h_{2}
\end{array}\right]^{T} \\
& \mathbf{m}_{4}=\left[\begin{array}{ll}
h_{2} & h_{1}
\end{array}\right]^{T} .
\end{aligned}
$$

Define $\alpha=\left|h_{1}+h_{2}\right|=\left[\alpha_{1}^{2}+2 \alpha_{1} \alpha_{2} \cos (\theta)+\alpha_{2}^{2}\right]^{1 / 2}$, where $\theta=\theta_{2}-\theta_{1}$. Define $\boldsymbol{\mu}_{i}$ to be the magnitude of $\left|\mathbf{m}_{i}\right|$. The magnitudes are

$$
\begin{aligned}
& \boldsymbol{\mu}_{1}=\left[\begin{array}{ll}
\alpha & 0
\end{array}\right]^{T} \\
& \boldsymbol{\mu}_{2}=\left[\begin{array}{ll}
0 & \alpha
\end{array}\right]^{T} \\
& \boldsymbol{\mu}_{3}=\left[\begin{array}{ll}
\alpha_{1} & \alpha_{2}
\end{array}\right]^{T} \\
& \boldsymbol{\mu}_{4}=\left[\begin{array}{ll}
\alpha_{2} & \alpha_{1}
\end{array}\right]^{T} .
\end{aligned}
$$

\section{RELAy RECEIVER Formulation}

Let $\mathbf{b}=\mathbf{b}_{1} \oplus \mathbf{b}_{2}$ be the network codeword. The job of the relay is to detect $\mathbf{b}$ and forward a remodulated version of it back to the end nodes. If an outer channel code is used, then the relay should perform soft-decision decoding of the network codeword prior to re-encoding and re-modulation. Soft-decision decoding requires that the relay compute the LLR of each network code bit $b$ according to

$$
\begin{aligned}
\Lambda(b) & =\log \frac{P(b=1 \mid \mathbf{y})}{P(b=0 \mid \mathbf{y})} \\
& =\log \frac{P\left(b_{1} \oplus b_{2}=1 \mid \mathbf{y}\right)}{P\left(b_{1} \oplus b_{2}=0 \mid \mathbf{y}\right)} \\
& =\log \frac{P\left(\left\{\mathbb{E}_{3} \cup \mathbb{E}_{4}\right\} \mid \mathbf{y}\right)}{P\left(\left\{\mathbb{E}_{1} \cup \mathbb{E}_{2}\right\} \mid \mathbf{y}\right)} \\
& =\log \frac{P\left(\mathbb{E}_{3} \mid \mathbf{y}\right)+P\left(\mathbb{E}_{4} \mid \mathbf{y}\right)}{P\left(\mathbb{E}_{1} \mid \mathbf{y}\right)+P\left(\mathbb{E}_{2} \mid \mathbf{y}\right)} .
\end{aligned}
$$

In the LNC system, the LLR's of $b_{1}$ and $b_{2}$ can be computed independently during the orthogonal time slots. The LLR of the signal sent from node $N_{i}$ to the relay is an expression in the form

$$
\Lambda\left(b_{i}\right)=\log \frac{P\left(b_{i}=1 \mid \mathbf{y}\right)}{P\left(b_{i}=0 \mid \mathbf{y}\right)}
$$

where $\mathbf{y}$ is the signal received during the time slot that node $N_{i}$ transmits. When the fading amplitudes $\alpha_{i}, i=1,2$, are known, then (12) is found using [7]

$$
\Lambda\left(b_{i}\right)=\log I_{0}\left(\frac{2 \sqrt{\mathcal{E}_{i}} \alpha_{i}\left|y_{2}\right|}{N_{0}}\right)-\log I_{0}\left(\frac{2 \sqrt{\mathcal{E}_{i}} \alpha_{i}\left|y_{1}\right|}{N_{0}}\right)
$$

where $I_{0}(\cdot)$ is the zeroth-order Bessel function of the first kind and $y_{1}$ and $y_{2}$ are the components of $\mathbf{y}$. If the fading amplitudes are not known, but have Rayleigh distributions, then (12) is found using [7]

$$
\Lambda\left(b_{i}\right)=\frac{\left(\mathcal{E}_{i} / N_{0}\right)^{2}}{1+\mathcal{E}_{i} / N_{0}}\left\{\left|y_{2}\right|^{2}-\left|y_{1}\right|^{2}\right\} .
$$

Once the individual LLR's from each end node are found using (13) or (14), the LLR of the LNC system's network codeword can then be found using the rules of log-likelihood arithmetic to be

$$
\begin{aligned}
\Lambda(b) & =\log \frac{e^{\Lambda\left(b_{1}\right)}+e^{\Lambda\left(b_{2}\right)}}{1+e^{\Lambda\left(b_{1}\right)+\Lambda\left(b_{2}\right)}} \\
& =\max *\left[\Lambda\left(b_{1}\right), \Lambda\left(b_{2}\right)\right]-\max *\left[0, \Lambda\left(b_{1}\right)+\Lambda\left(b_{2}\right)\right]
\end{aligned}
$$

where $\max *[x, y]=\log \left(e^{x}+e^{y}\right)$.

In the PNC system, it is not sensible to compute $\Lambda\left(b_{1}\right)$ and $\Lambda\left(b_{2}\right)$ separately. Instead, use (11) and assume that the four events are equally likely along with Bayes rule to obtain

$$
\begin{aligned}
\Lambda(b)= & \log \left[p\left(\mathbf{y} \mid \mathbb{E}_{3}\right)+p\left(\mathbf{y} \mid \mathbb{E}_{4}\right)\right] \\
& -\log \left[p\left(\mathbf{y} \mid \mathbb{E}_{1}\right)+p\left(\mathbf{y} \mid \mathbb{E}_{2}\right)\right] .
\end{aligned}
$$

The computation of each $p\left(\mathbf{y} \mid \mathbb{E}_{i}\right)$ by the PNC relay receiver given various levels of channel state information is the subject of the remainder of this section.

\section{A. Coherent Reception}

When the mean vector $\mathbf{m}_{i}$ is known, then we may write $p\left(\mathbf{y} \mid \mathbb{E}_{i}\right)=p\left(\mathbf{y} \mid \mathbf{m}_{i}\right)$ and the conditional probability density function (pdf) is:

$$
p\left(\mathbf{y} \mid \mathbf{m}_{i}\right)=\left(\frac{1}{\pi N_{0}}\right)^{2} \exp \left\{-\frac{1}{N_{0}}\left\|\mathbf{y}-\mathbf{m}_{i}\right\|^{2}\right\} .
$$

The coherent receiver computes each of the $p\left(\mathbf{y} \mid \mathbb{E}_{i}\right)$ required by (16) by substituting the corresponding $\mathbf{m}_{i}$ defined by (9) into (17).

\section{B. Noncoherent Reception with CSI}

Suppose that the receiver does not know the individual phases $\theta_{1}, \theta_{2}$, but it has channel state information (CSI) by knowing the values of the four magnitude vectors $\boldsymbol{\mu}_{i}$. Given event $\mathbb{E}_{i}, i=1,2,3,4$, the $k^{\text {th }}$ component, $k=1,2$, of the received signal vector may be expressed as:

$$
y_{k}=\mu_{i, k} e^{j \phi_{i, k}}+n_{k}
$$

where $\phi_{i, k}$ is an unknown phase. The conditional pdf given $\boldsymbol{\mu}_{i}$ is found by marginalizing over the unknown $\left\{\phi_{i, 1}, \phi_{i, 2}\right\}$

$$
p\left(\mathbf{y} \mid \boldsymbol{\mu}_{i}\right)=\int_{0}^{2 \pi} \int_{0}^{2 \pi} p\left(\phi_{i, 1}, \phi_{i, 2}\right) p\left(\mathbf{y} \mid \mathbf{m}_{i}\right) d \phi_{i, 1} d \phi_{i, 2} .
$$

For Rayleigh fading, $\theta_{1}$ and $\theta_{2}$ are i.i.d. uniform over $[0,2 \pi)$. Using the facts that each $h_{k}=\alpha_{k} e^{j \theta_{k}}, k=1,2$, can be expressed as a complex-valued, circularly-symmetric Gaussian random variable and that the addition of both of these independent random variables gives another random variable of the same type, we conclude that the phase of the latter is uniformly distributed over $[0,2 \pi)$. Therefore, $\phi_{i, 1}$ and $\phi_{i, 2}$ in 
(18) and (19) are i.i.d. uniform over $[0,2 \pi)$. The amplitude $\alpha$ is independent of the phase, and $E\left[\alpha^{2}\right]=E\left[\alpha_{1}^{2}\right]+E\left[\alpha_{2}^{2}\right]$. The conditional pdf becomes

$$
\begin{aligned}
p\left(\mathbf{y} \mid \boldsymbol{\mu}_{i}\right)= & \frac{1}{2 \pi^{2} N_{0}} \int_{0}^{2 \pi} \exp \left\{-\frac{\left|y_{1}-\mu_{i, 1} e^{j \phi_{i, 1}}\right|^{2}}{N_{0}}\right\} d \phi_{i, 1} \\
& \times \frac{1}{2 \pi^{2} N_{0}} \int_{0}^{2 \pi} \exp \left\{-\frac{\left|y_{2}-\mu_{i, 2} e^{j \phi_{i, 2}}\right|^{2}}{N_{0}}\right\} d \phi_{i, 2}
\end{aligned}
$$

where

$$
\begin{aligned}
& \frac{1}{2 \pi} \int_{0}^{2 \pi} \exp \left\{-\frac{\left|y_{k}-\mu_{i, k} e^{j \phi_{i, k}}\right|^{2}}{N_{0}}\right\} d \phi_{i, k} \\
& =\exp \left\{-\frac{\left|y_{k}\right|^{2}+\mu_{i, k}^{2}}{N_{0}}\right\} I_{0}\left(\frac{2\left|y_{k}\right| \mu_{i, k}}{N_{0}}\right) .
\end{aligned}
$$

Substituting (21) into (20),

$$
p\left(\mathbf{y} \mid \boldsymbol{\mu}_{i}\right)=\beta \prod_{k=1}^{2} \exp \left\{-\frac{\mu_{i, k}^{2}}{N_{0}}\right\} I_{0}\left(\frac{2\left|y_{k}\right| \mu_{i, k}}{N_{0}}\right)
$$

where

$$
\beta=\left(\frac{1}{\pi N_{0}}\right)^{2} \exp \left\{-\left(\frac{\left|y_{1}\right|^{2}+\left|y_{2}\right|^{2}}{N_{0}}\right)\right\}
$$

which is common to all four events and will therefore cancel in the LLR (16).

Define

$$
\begin{aligned}
\gamma_{i} & =\frac{\alpha_{i}^{2}}{N_{0}}, \quad i=\{1,2\} \\
\gamma & =\frac{\alpha^{2}}{N_{0}}
\end{aligned}
$$

Note that since $E\left[\alpha^{2}\right]=E\left[\alpha_{1}^{2}\right]+E\left[\alpha_{2}^{2}\right]$,

$$
E[\gamma]=E\left[\gamma_{1}\right]+E\left[\gamma_{2}\right]
$$

For each event $\mathbb{E}_{i}$, substitute the $p\left(\mathbf{y} \mid \boldsymbol{\mu}_{i}\right)$ given in (22) with the $\mu_{i, k}$ given by (10) as the corresponding $p\left(\mathbf{y} \mid \mathbb{E}_{i}\right)$ in (16). This results in

$$
\begin{aligned}
\Lambda(b)= & \log \left[e^{-\gamma_{1}} I_{0}\left(\frac{2\left|y_{1}\right|}{\sqrt{N_{0} / \gamma_{1}}}\right) e^{-\gamma_{2}} I_{0}\left(\frac{2\left|y_{2}\right|}{\sqrt{N_{0} / \gamma_{2}}}\right)\right. \\
& \left.+e^{-\gamma_{2}} I_{0}\left(\frac{2\left|y_{1}\right|}{\sqrt{N_{0} / \gamma_{2}}}\right) e^{-\gamma_{1}} I_{0}\left(\frac{2\left|y_{2}\right|}{\sqrt{N_{0} / \gamma_{1}}}\right)\right] \\
& -\log \left[e^{-\gamma} I_{0}\left(\frac{2\left|y_{1}\right|}{\sqrt{N_{0} / \gamma}}\right)+e^{-\gamma} I_{0}\left(\frac{2\left|y_{2}\right|}{\sqrt{N_{0} / \gamma}}\right)\right] .
\end{aligned}
$$

Define $F(x)=\log \left[I_{0}(x)\right]$; then the LLR becomes

$$
\begin{aligned}
\Lambda(b)= & \gamma-\gamma_{1}-\gamma_{2} \\
& +\max *\left[F\left(\frac{2\left|y_{1}\right|}{\sqrt{N_{0} / \gamma_{1}}}\right)+F\left(\frac{2\left|y_{2}\right|}{\sqrt{N_{0} / \gamma_{2}}}\right),\right. \\
& \left.F\left(\frac{2\left|y_{1}\right|}{\sqrt{N_{0} / \gamma_{2}}}\right)+F\left(\frac{2\left|y_{2}\right|}{\sqrt{N_{0} / \gamma_{1}}}\right)\right] \\
& -\max *\left[F\left(\frac{2\left|y_{1}\right|}{\sqrt{N_{0} / \gamma}}\right), F\left(\frac{2\left|y_{2}\right|}{\sqrt{N_{0} / \gamma}}\right)\right] .
\end{aligned}
$$

The function $F(x)$ may be efficiently computed through a piecewise polynomial fit that returns precise answers over a wide range of the argument:

$$
\begin{gathered}
F(x)=\log \left[I_{0}(x)\right]= \\
\begin{cases}0.22594 x^{2}+0.012495 x-0.0011272 & 0<x \leq 1 \\
0.12454 x^{2}+0.21758 x-0.10782 & 1<x \leq 2 \\
0.028787 x^{2}+0.63126 x-0.56413 & 2<x \leq 5 \\
0.003012 x^{2}+0.88523 x-1.2115 & 5<x \leq 15 \\
0.00053203 x^{2}+0.95304 x-1.6829 & 15<x \leq 30 \\
0.00013134 x^{2}+0.97674 x-2.0388 & 30<x \leq 60 \\
0.9943 x-2.6446 & 60<x \leq 120 \\
0.99722 x-3.0039 & 120<x \leq 500 \\
0.99916 x-3.6114 & x>500 .\end{cases}
\end{gathered}
$$

If $\alpha_{1}$ and $\alpha_{2}$ are known, but $\alpha$ is not, then (25) suggests that a reasonable approximation is

$$
\gamma \approx \gamma_{1}+\gamma_{2}
$$

and the LLR is found by substituting (29) into (27).

\section{Noncoherent Reception without CSI}

Now suppose that besides not knowing the phases $\theta_{1}, \theta_{2}$, the receiver does not know the magnitude vectors $\boldsymbol{\mu}_{i}$ either. Therefore, the relay must operate without any channel state information except for the average energies $\mathcal{E}_{1}, \mathcal{E}_{2}$. When the magnitudes $\boldsymbol{\mu}_{i}$ are not known, then the conditional pdf is found by marginalizing (22) over the unknown $\left\{\mu_{i, 1}, \mu_{i, 2}\right\}$

$$
p\left(\mathbf{y} \mid \mathbb{E}_{i}\right)=\int_{0}^{\infty} \int_{0}^{\infty} p\left(\mu_{i, 1}, \mu_{i, 2}\right) p\left(\mathbf{y} \mid \boldsymbol{\mu}_{i}\right) d \mu_{i, 1} d \mu_{i, 2}
$$

As discussed below (19), $h_{1}$ and $h_{2}$ are independent complexvalued, circularly-symmetric Gaussian variables, and therefore $h=h_{1}+h_{2}$ is also a complex-valued, circularly-symmetric Gaussian variable. Thus, the amplitudes $\left\{\alpha_{1}, \alpha_{2}, \alpha\right\}$ of the three variables $\left\{h_{1}, h_{2}, h\right\}$ have Rayleigh distributions. As shown in (10), each nonzero value of $\mu_{i, k}$ is either $\alpha_{1}, \alpha_{2}$, or $\alpha$. Since each of these variables is Rayleigh, the marginal pdf of each nonzero $\mu_{i, k}$ is

$$
p\left(\mu_{i, k}\right)=\frac{2 \mu_{i, k}}{\mathcal{E}_{i, k}} \exp \left\{-\frac{\mu_{i, k}}{\mathcal{E}_{i, k}}\right\}, \quad \mu_{i, k} \geq 0
$$

where $\mathcal{E}_{i, k}=E\left[\mu_{i, k}^{2}\right]$. Note that $\mathcal{E}_{i, k}=\mathcal{E}_{1}$ when $\mu_{i, k}=\alpha_{1}$, $\mathcal{E}_{i, k}=\mathcal{E}_{2}$ when $\mu_{i, k}=\alpha_{2}$, and $\mathcal{E}_{i, k}=\mathcal{E}_{1}+\mathcal{E}_{2}$ when $\mu_{i, k}=$ $\alpha_{1}+\alpha_{2}$. Using (31), the $\mu_{i, k}$ given by (10), and the fact that $\alpha_{1}$ and $\alpha_{2}$ are independent, the joint pdf $p\left(\mu_{i, 1}, \mu_{i, 2}\right)$ of each event $\mathbb{E}_{i}$ may be found. Marginalizing each $p\left(\mathbf{y} \mid \boldsymbol{\mu}_{i}\right)$ with respect to the corresponding $p\left(\mu_{i, 1}, \mu_{i, 2}\right)$ and substituting into 
(16) yields

$$
\begin{aligned}
& \Lambda(b)=\log \left[\frac{\xi_{1} \xi_{2}}{\xi N_{0}}\right] \\
& +\log \left[\exp \left\{-\frac{\left|y_{1}\right|^{2}}{2 \xi}-\frac{\left|y_{2}\right|^{2}}{2 N_{0}}\right\}+\exp \left\{-\frac{\left|y_{1}\right|^{2}}{2 N_{0}}-\frac{\left|y_{2}\right|^{2}}{2 \xi}\right\}\right] \\
& -\log \left[\exp \left\{-\frac{\left|y_{1}\right|^{2}}{2 \xi_{1}}-\frac{\left|y_{2}\right|^{2}}{2 \xi_{2}}\right\}+\exp \left\{-\frac{\left|y_{1}\right|^{2}}{2 \xi_{2}}-\frac{\left|y_{2}\right|^{2}}{2 \xi_{1}}\right\}\right]
\end{aligned}
$$

where

$$
\begin{aligned}
\xi_{1} & =\mathcal{E}_{1}+N_{0} \\
\xi_{2} & =\mathcal{E}_{2}+N_{0} \\
\xi & =\mathcal{E}_{1}+\mathcal{E}_{2}+N_{0} .
\end{aligned}
$$

In terms of $\max *(\cdot)$, (32) may be expressed as

$$
\begin{aligned}
& \Lambda(b)=\log \left[\frac{\xi_{1} \xi_{2}}{\xi N_{0}}\right] \\
& +\max *\left\{-\frac{1}{2}\left(\frac{\left|y_{1}\right|^{2}}{\xi}+\frac{\left|y_{2}\right|^{2}}{N_{0}}\right),-\frac{1}{2}\left(\frac{\left|y_{1}\right|^{2}}{N_{0}}+\frac{\left|y_{2}\right|^{2}}{\xi}\right)\right\} \\
& -\max *\left\{-\frac{1}{2}\left(\frac{\left|y_{1}\right|^{2}}{\xi_{1}}+\frac{\left|y_{2}\right|^{2}}{\xi_{2}}\right),-\frac{1}{2}\left(\frac{\left|y_{1}\right|^{2}}{\xi_{2}}+\frac{\left|y_{2}\right|^{2}}{\xi_{1}}\right)\right\} .
\end{aligned}
$$

\section{Performance without an Outer Code}

We now demonstrate the simulated performance of the proposed PNC system and the relay receivers derived in Section III. We initially consider a system that does not use an outer error-correcting code, and thus $\mathbf{b}_{i}=\mathbf{u}_{i}, i=1,2$. In the simulation, each end node generates a random message and transmits it to the relay using binary CPFSK modulation.

With the LNC system, the two nodes transmit their messages in orthogonal time slots and the relay receiver first generates the individual LLR's during each time slot using either (13) or (14), and then the two LLR's are combined using (15). When there is no outer error-correcting code, performance using (13) is approximately the same as that using (14). A bit error is declared at the relay whenever a hard decision using (15) results in an erroneous decision on the corresponding bit of the network codeword $\mathbf{b}$. Such an error will usually occur if one of the two bits $b_{1}, b_{2}$ is received incorrectly, and therefore the error rate of the LNC system is approximately $P_{b} \approx 2 p(1-p)$ where $p$ is the bit error rate of noncoherent binary FSK modulation [8].

With the PNC system, the two nodes transmit simultaneously, and the relay receiver computes the LLR using (27) when the magnitudes $\boldsymbol{\mu}_{i}$ are known or (34) when they are not. A hard decision is made on the LLR and a bit error is declared if the estimate of the corresponding network codeword bit $b$ is incorrect.

For both systems, after the hard decision on $b$ is made, the relay will remodulate the network code bit using binary CPFSK modulation and broadcast it to both end nodes. Because the relay-broadcast phase is the same for both the LNC and PNC systems, we only focus on the performance at the

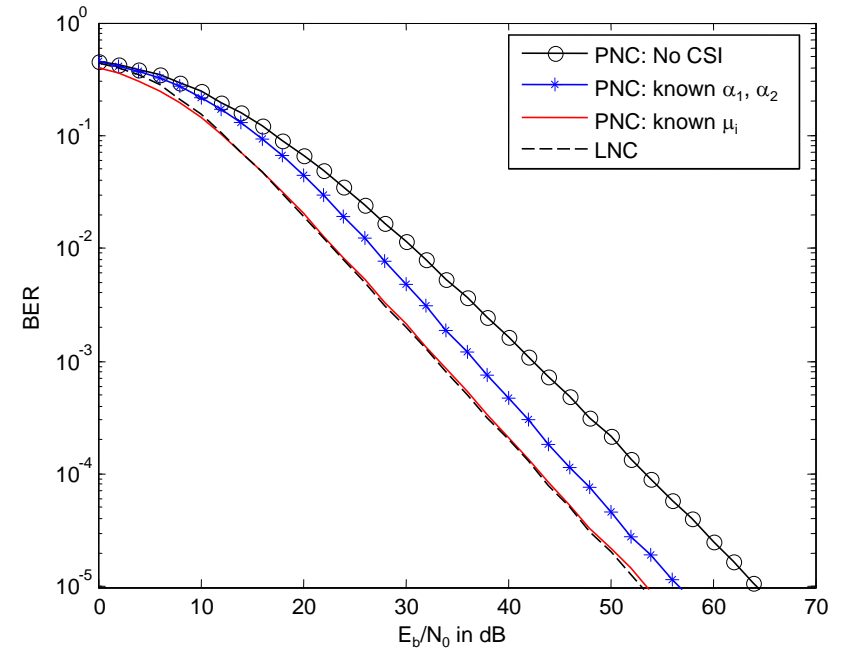

Fig. 2. Bit error rate at the relay in Rayleigh fading when PNC and LNC is used and $\mathcal{E}_{2}=\mathcal{E}_{1}$. Depending on the amount of channel state information that is available, the PNC system will use one of three different relay receivers.

relay. In particular, we determine the probability that the relay makes an erroneous decision on the network codeword $\mathbf{b}$.

Initially, we set the average received energy to be the same over both channels, i.e. $\mathcal{E}_{2}=\mathcal{E}_{1}=\mathcal{E}_{s}=\mathcal{E}_{b}$. Fig. 2 shows the performance of the LNC and PNC systems in Rayleigh fading with equal energy signals. As anticipated, the LNC system offers the best performance, which is approximately $3 \mathrm{~dB}$ worse than a standard binary CPFSK system with noncoherent detection (the loss relative to conventional CPFSK is due to the fact that both bits must usually be received correctly).

Three curves for the PNC system are shown in Fig. 2, corresponding to receivers that exploit different amounts of available channel state information. The best performance is achieved using a receiver implemented with (27), which requires knowledge of $\alpha_{1}, \alpha_{2}$, and $\alpha$. The performance of the PNC system implemented with (27) is only about $0.25 \mathrm{~dB}$ worse than that of the LNC system. The worst performance is achieved using a receiver implemented using (34), which does not require knowledge of the fading amplitudes. The loss due to using (34) instead of (27) is about $10 \mathrm{~dB}$, suggesting that estimating the fading amplitudes at the relay is advantageous.

While it may be feasible to estimate $\alpha_{1}$ and $\alpha_{2}$, estimating $\alpha$ may prove to be more difficult because it will depend on not only the individual fading amplitudes, but also on the phase difference between the two channels. Since the phase difference might change more quickly than the individual amplitudes, it might not be practical to estimate $\alpha$. If that is the case, then the approximation given by (29) can be used in place of the actual value of $\gamma$. The performance using this technique is also shown in Fig. 2 and shows a loss of about $3 \mathrm{~dB}$ with respect to the known- $\boldsymbol{\mu}_{i}$ system which requires knowledge of $\alpha$.

The performance of PNC is sensitive to the balance of power received over the two channels. Performance is best when $\mathcal{E}_{1}=\mathcal{E}_{2}$. In order to evaluate how robust the PNC relay receivers are to an imbalance of power, the simulations were 


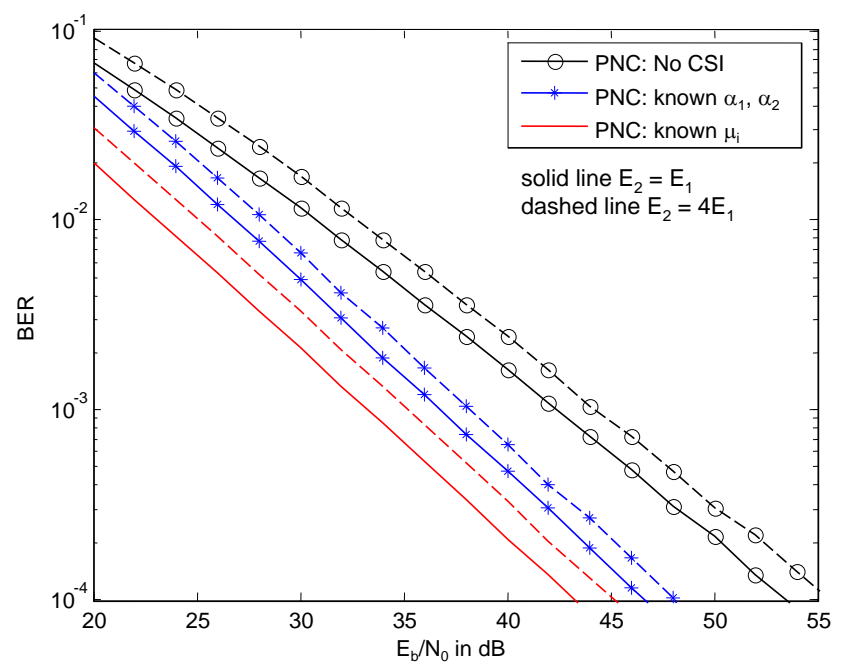

Fig. 3. Bit error rate at the relay in Rayleigh fading when PNC is used with three different receivers and $\mathcal{E}_{2}=4 \mathcal{E}_{1}$ (solid line) or $\mathcal{E}_{2}=\mathcal{E}_{1}$ (dashed line).

repeated with $\mathcal{E}_{2}=4 \mathcal{E}_{1}$, while keeping $\mathcal{E}_{b}=\mathcal{E}_{s}=\left(\mathcal{E}_{1}+\mathcal{E}_{2}\right) / 2$. These results are shown in Fig. 3 for the three receiver formulations that were considered in the previous figure. When the power is imbalanced in this way, there is a loss of about $2 \mathrm{~dB}$. However, the loss is the same for all three receiver implementations, suggesting that they are robust to an imbalance of power.

\section{Performance with Outer Turbo Code}

Consider the performance of the proposed PNC system with an outer turbo code. Each end node $N_{i}$ encodes its length $K$ message $\mathbf{u}_{i}$ into a length $L=K / r$ codeword $\mathbf{b}_{i}$ using a common rate-r turbo code $\mathcal{C}$. The network-coded turbo codeword is $\mathbf{b}=\mathbf{b}_{1}+\mathbf{b}_{2}$. Since the turbo code is linear, $\mathbf{b} \in \mathcal{C}$, and the message $\mathbf{u}$ corresponding to $\mathbf{b}$ satisfies $\mathbf{u}=\mathbf{u}_{1}+\mathbf{u}_{2}$.

For both the LNC and PNC systems, each end node modulates its turbo codeword using CPFSK modulation and transmits it to the relay. In the LNC system, the two turbo codewords are sent in orthogonal timeslots, whereas in the PNC system, they are sent at the same time. The relay computes the LLR of the network codeword $\Lambda(\mathbf{b})$, which is passed through a turbo decoder to generate an estimate $\hat{\mathbf{u}}$ of $\mathbf{u}$. If any bit of $\hat{\mathbf{u}}$ does not agree with the same bit in $\mathbf{u}$, then a bit error is logged at the relay.

In the LNC system, the LLR of each bit is computed by using either (13) or (14) and then the two LLR's are substituted into (15) to determine the LLR of each bit of the networkcoded turbo codeword. When the relay receiver has channel state information in the form of fading-amplitude estimates, it uses (13). If the receiver does not know the instantaneous values of the fading amplitudes, but knows $\mathcal{E}_{1}$ and $\mathcal{E}_{2}$, then it uses (14). Unlike the uncoded case, performance of the LNC system is slightly better with CSI than without.

With the PNC system, the two nodes transmit simultaneously and the relay receiver computes the LLR of the networkcoded turbo codeword using (27) when the magnitudes $\boldsymbol{\mu}_{i}$ are known or (34) when they are not.

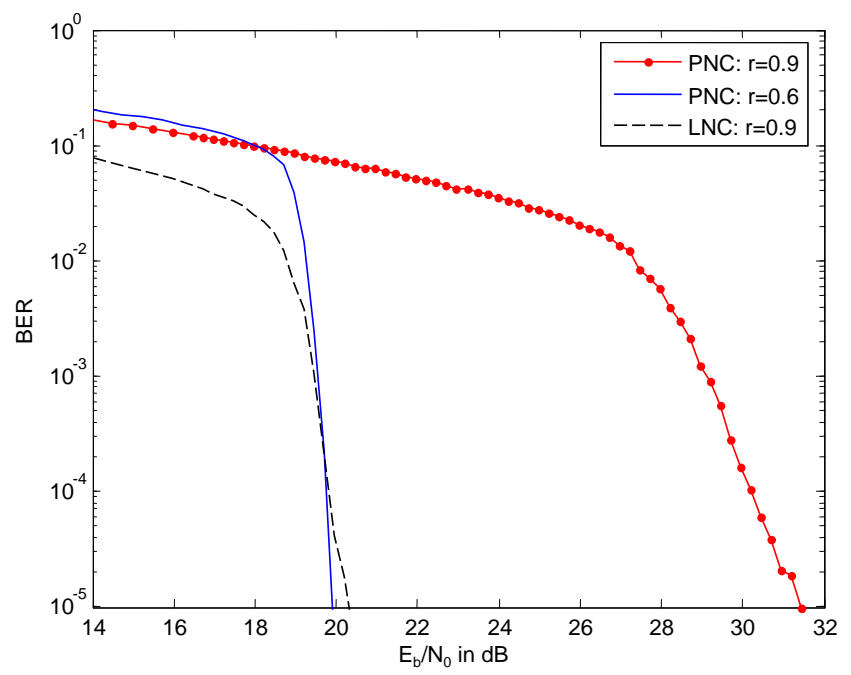

Fig. 4. Bit error rate at the relay in Rayleigh fading with a turbo code assuming that channel state information is not available.

In both systems, the estimated message $\hat{\mathbf{u}}$ is turbo encoded at the relay to produce a new turbo-coded network codeword $\hat{\mathbf{b}}$ which is broadcast to both end nodes. Each end node proceeds to decode this codeword to produce its own estimate $\tilde{\mathbf{u}}$ of the network-coded message, which it adds to its own message, thereby revealing an estimate of the other end node's transmitted message. The turbo codeword broadcast by the relay does not necessarily need to be encoded at the same rate that was used to encode the messages at the end nodes. In the case of a LNC system, the performance from the end nodes to the relay is about the same as the performance from the relay to the end nodes, and thus there is no compelling reason to use different code rates. On the other hand, the performance of the PNC system is much more asymmetric. The performance from the end nodes to the relay tends to be worse than the performance from the relay to the end nodes, and thus it is sensible to use a stronger code on the first phase (end nodes to relay) than on the second phase (relay to end nodes). When asymmetric code rates are used, then the duration of the two transmission phases must necessarily be different in order to maintain a constant symbol rate and, hence, a constant bandwidth.

We first consider the case that no channel state information is available at the relay. For the LNC system, the demodulator is implemented using (14), while for the PNC system it is implemented using (34). We use the turbo code that has been standardized in the UMTS third-generation cellular system [9]. This code was selected because of its widespread adoption and its ability to support a wide range of code rates due to its ratematching algorithm. The baseline system uses length $K=$ 4500 messages and length $N=5000$ turbo codewords; thus, the rate is $r=4500 / 5000=0.9$. The bit error rate observed at the relay using the rate $r=0.9$ code in the LNC system is shown in Fig. 4, where $\mathcal{E}_{b}=\mathcal{E}_{s} / r$. The same rate $r=0.9$ code was also used in the PNC system, and the performance is also shown in Fig. 4. Note that the PNC system requires over $10 \mathrm{~dB}$ more energy than the LNC system to achieve a 


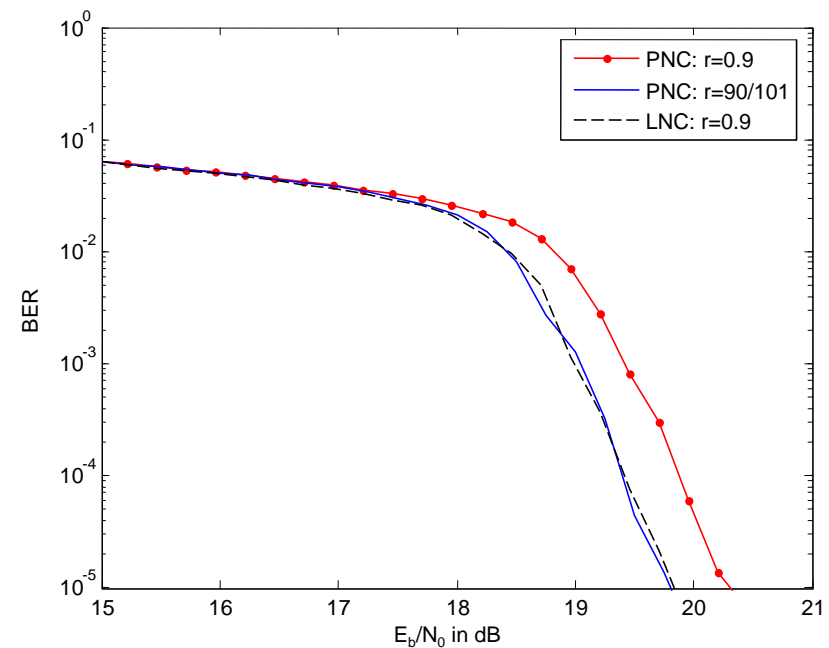

Fig. 5. Bit error rate at the relay in Rayleigh fading with a turbo code assuming that channel state information is available.

bit error rate of $10^{-4}$. However, the PNC system has a higher throughput than the LNC system. For instance, if the relaybroadcast phase also uses a rate $r=0.9$ turbo code, then the throughput of the PNC system would be $50 \%$ higher than that of the LNC system because it would only need two time slots compared to the LNC system's three time slots.

The loss in energy efficiency due to using PNC can be recovered by using a more powerful, lower-rate turbo code at the end nodes (the relay can still broadcast using the rate 0.9 turbo code). As an example, the rate $r=4500 / 7500=0.6$ turbo code could be used to encode the messages at each end node. The BER at the relay using this lower rate code in the PNC system is shown in Fig. 4 and is approximately the same as the BER of the LNC system with the rate $r=0.9$ code. To compare the throughput of LNC and PNC, consider that in both cases, 9,000 information bits are exchanged $(4,500$ from each end node). Let $T$ be the time duration (in seconds) required to transmit 5,000 code bits. For each message exchange, the LNC system requires three full slots of duration $T$ second. Thus, the throughput of LNC is $9000 / 3 T=3000 / T$ bits per second (bps). The PNC system only requires 2 slots, with the the first slot of duration $1.5 \mathrm{~T}(7,500$ code bits) and the second of duration $T$. Thus, the throughput of PNC is $9000 / 2.5 T=3600 / T$ bps. As $(3600 / T) /(3000 / T)=1.2$, we can conclude that the throughput of the PNC system is $20 \%$ higher than that of the corresponding LNC system.

Next, we consider the case that channel state information is available at the relay in the form of fading-amplitude estimates. For the LNC system, the demodulator is implemented using (13) along with knowledge of $\alpha_{1}$ and $\alpha_{2}$, while for the PNC system it is implemented using (27) with knowledge of $\alpha_{1}, \alpha_{2}$, and $\alpha$. The BER at the relay of the two systems with the $r=4500 / 5000=0.9$ code is shown in Fig. 5 . By comparing with the results of Fig. 4, it is observed that the LNC system gains about $0.4 \mathrm{~dB}$ in energy efficiency when the relay receiver exploits knowledge of the channel gains. In contrast, the performance gain of PNC is much more dramatic. In particular, the PNC system gains nearly $10 \mathrm{~dB}$ in energy efficiency when the relay receiver uses the known channel gains. At a BER of $10^{-4}$, the energy efficiency of the PNC system is only about $0.5 \mathrm{~dB}$ worse than the LNC system. In order to recover this slight loss in energy efficiency, a slightly lower code rate could be used. In particular, using a rate $r=4500 / 5050 \approx 0.89$ code recovers this loss. As shown in Fig. 5, the performance of PNC system with the rate $r \approx 0.89$ is approximately the same as the performance of the LNC system with the rate $r=0.9$ code. The LNC system requires three slots each of duration $T$ for the exchange of 9000 information bits, and thus has a throughput of $9000 /(3 T)=3000 / T$ bps. The PNC system requires two slots, the first of duration 1.01T and the second of duration $\mathrm{T}$, and has a throughput of $9000 /(2.01 T) \approx 4,477 / T$ bps, which is $49.25 \%$ higher than that of the LNC system.

\section{CONCLUSION}

Physical-layer network coding is an effective way for a pair of terminals to exchange information via a relay. However, the usual assumptions of phase-synchronous operation and coherent reception are not practical. The noncoherent relay receiver derived in this paper does not require phase synchronism, thereby making physical-layer network coding more feasible. When coupled with a turbo code, the noncoherent physicallayer network coding system offers better throughput than the corresponding link-layer network coding system. The example given in this paper shows that the throughput of PNC can be $20 \%$ more than that of LNC when the relay receiver only knows the channel statistics and nearly 50\% more when it knows the fading amplitudes. The excellent performance when the fading amplitudes are known serves as a motivation for studying algorithms for estimating these fading amplitudes, which we leave as an open problem.

\section{REFERENCES}

[1] R. Ahlswede, N. Cai, S. Li, and R. Yeung, "Network information flow," IEEE Trans. Inform. Theory, vol. 46, pp. 1204-1216, July 2000.

[2] S. Zhang, S. C. Liew, and P. P. Lam, "Physical-layer network coding," in Proc. Int. Conf. on Mobile Computing and Networking (MOBICOM), Los Angeles, CA, Sept. 2006, pp. 358-365.

[3] S. Zhang and S.-C. Liew, "Channel coding and decoding in a relay system operated with physical-layer network coding," IEEE J. Select. Areas Commun., vol. 27, pp. 788-796, June 2009.

[4] E. Peh, Y. Liang, and Y. L. Guan, "Power control for physical-layer network coding in fading environments," in Proc. IEEE Personal Indoor and Mobile Radio Commun. Conf., Cannes, France, Sept. 2008, pp. 1-5.

[5] S. Katti, H. Rahul, W. Hu, D. Katabi, M. Medard, and J. Crowcroft, "XORs in the air: Practical wireless network coding," IEEE/ACM Trans. Networking, vol. 16, pp. 497-510, June 2008.

[6] P. Popovski and H. Yomo, "Wireless network coding by amplify-andforward for bi-directional traffic flows," IEEE Commun. Lett, vol. 11, pp. 16-18, Jan. 2007.

[7] M. C. Valenti and S. Cheng, "Iterative demodulation and decoding of turbo coded $M$-ary noncoherent orthogonal modulation," IEEE J. Select. Areas Commun., vol. 23, pp. 1738-1747, Sept. 2005.

[8] J. G. Proakis and M. Salehi, Digital Communications, 5th ed. New York, NY: McGraw-Hill, Inc., 2008.

[9] European Telecommunications Standards Institute, "Universal mobile telecommunications system (UMTS): Multiplexing and channel coding (FDD)," 3GPP TS 25.212 version 3.4.0, Sept. 23, 2000. 